Journal of Universal Mathematics

VOL.2 No.1 PP.16-21 (2019)

ISSN-2618-5660

\title{
AN APPLICATION OF PHASE-TYPE DISTRIBUTIONS IN A RELIABILITY SHOCK MODEL
}

\author{
MUSTAFA TEKIN AND SERKAN ERYILMAZ
}

\begin{abstract}
Phase-type distributions have been found to be very useful in reliability, queueing theory, and some other operational research applications. In this work, we define a new mixed shock model and study lifetime properties of the corresponding system using phase-type distributions. The system under concern fails upon the occurrence of $k$ consecutive shocks of size at least $d_{1}$ or a single large shock of size at least $d_{2}, d_{1}<d_{2}$. In particular, using closure properties of phase-type distributions, we obtain survival function and mean time to failure of the system. Optimal replacement time is also studied.
\end{abstract}

\section{INTRODUCTION}

Shock models have attracted much attention in applied probability. In any kind of a shock model, the system is subject to shocks over time. The shocks occur at random times and the magnitudes of the shocks are also assumed to be random. Various shock models have been defined and studied in the literature. The most well-known models are the extreme shock model, the cumulative shock model, the delta-shock model, and the run shock model. In the extreme shock model, the system fails upon the occurrence of one large critical shock (Shanthikumar and Sumita (1983), Gut and Husler (1999), Cha and Finkelstein (2011), Cirillo and Husler (2011)). Two new generalizations of the extreme shock model have been studied by Bozbulut and Eryilmaz (2018). In the run shock model, the system fails upon the occurrence of $\mathrm{k}$ consecutive shocks above a critical level (see, e.g. Mallor and Omey (2001)). Mixed shock models have also been considered in the literature. In these type of shock models, the system has two failure criteria.

In shock models, the lifetime of the system can be represented as a random sum of random variables. Let $T$ denote the systems lifetime, then we have $T=\sum_{i=1}^{N} X_{i}$, where the sequence of $X_{i}$ represents the times between shocks and the random variable $N$ denotes the number of shocks until the failure of the system. If $N$ and $X_{i}$ have phase-type distributions, then the lifetime random variable $T$ has also a phase type distribution. This is a very useful closure property of phase-type distributions.

In this paper, we study a new mixed shock model using phase-type distributions. According to the model, the system fails if either a run of $k$ consecutive shocks of

Date: Review January 2, 2019, accepted January 26, 2019.

2000 Mathematics Subject Classification. Primary 90B25.

Key words and phrases. Reliability, Shock models, Phase type distribution. 
size at least $d_{1}$ occurs or a single shock of size at least $d_{2}$ occurs, where $d_{1}$ and $d_{2}$ are two fixed thresholds such that $d_{1}<d_{2}$ (Eryilmaz and Tekin (2018)). Using properties of phase-type distributions, we study optimal replacement time for the system under concern.

\section{THE Model}

Eryilmaz and Tekin (2018) defined and studied a mixed shock model which combines extreme and run shock models. Consider a system that is subject to a sequence of shocks over time. Let $X_{i}$ denote the time when the first shock occurs, and $X_{i}$ be the interarrival time between the $(i-1)$-th and $i$-th shock $i \geq 2 . Y_{i}$ to be the magnitude of the $i$-th shock, $i \geq 1$. Let us fix two critical values $d_{1}$ and $d_{2}$ such that $d_{1}<d_{2}$. The system fails upon the occurrence of $k$ consecutive shocks of size at least $d_{1}$ or a single large shock of size at least $d_{2}$. If $N$ denotes the number of shocks until $k$ consecutive shocks above or equal to $d_{1}$ or a single shock of size at least $d_{2}$, then the failure time of the system can be expressed as

$$
T=\sum_{i=1}^{N} X_{i}
$$

Throughout the paper, the random variables $X_{1}, X_{2}, \ldots$ are assumed to have phasetype distributions. As shown by Eryilmaz and Tekin (2018), the random variable $N$ has also a phase-type distribution. A discrete phase type distribution is the distribution of the time to absorption in an absorbing Markov chain, and its probability mass function (pmf) has the following form

$$
P(N=n)=\mathbf{a Q}^{n-1} \mathbf{u}^{\prime}
$$

for $n=1 ; 2, \ldots$ In $(2.2), \mathbf{Q}=\left(q_{i j}\right)_{k \times k}$ is a matrix which includes the transition probabilities among the $k$ transient states, and $\mathbf{u}^{\prime}=(\mathbf{I}-\mathbf{Q}) \mathbf{e}^{\prime}$ is a vector which includes the transition probabilities from transient states to the absorbing state, $\mathbf{a}=$ $\left(a_{1}, \ldots, a_{k}\right)$ with $\sum_{i=1}^{k} a_{i}=1$, and $\mathbf{I}$ is the identity matrix (see, e.g. Neuts (1981)). The matrix $\mathbf{Q}$ must satisfy the condition that $\mathbf{I}-\mathbf{Q}$ is nonsingular. The survival function and the probability generating function (pgf) of $N$ can be respectively computed from

$$
P(N>n)=\mathbf{a Q}^{x} \mathbf{e}^{\prime}
$$

and

$$
\phi(z)=1-\mathbf{a e}^{\prime}+\mathbf{a} z(\mathbf{I}-z \mathbf{Q})^{-1} \mathbf{u}^{\prime}
$$

where $\mathbf{e}=(1, \ldots, 1)_{1 \times k}$. The expected value of $N$ is

$$
E(N)=\mathbf{a}(\mathbf{I}-\mathbf{Q})^{-1} \mathbf{e}^{\prime}
$$

Eryilmaz and Tekin (2018) has obtained the following phase representation for the random variable $N$. 
Lemma 2.1. Let $p_{1}=P\left\{d_{1}<Y_{i}<d_{2}\right\}$ and $p_{2}=P\left\{Y_{i}>d_{2}\right\}$ for $i=1,2, \ldots$, then $N \sim P H_{d}(\mathbf{a}, \mathbf{Q})$ with $\mathbf{a}=(1, \ldots 0)$,

$$
\mathbf{Q}=\left[\begin{array}{ccccc}
1-p_{1}-p_{2} & p_{1} & 0 & \cdots & 0 \\
1-p_{1}-p_{2} & 0 & p_{1} & \cdots & 0 \\
\vdots & & & \ddots & \\
1-p_{1}-p_{2} & 0 & 0 & \cdots & p_{1} \\
1-p_{1}-p_{2} & 0 & 0 & \cdots & 0
\end{array}\right]_{k \times k}
$$

and $\mathbf{u}=\left(p_{2}, \ldots, p_{2}, p_{1}+p_{2}\right)$.

The following results are immediate from Lemma 2.1.

Corollary 2.2. Using Lemma (2.1) and Equation (2.5), the expected value of $N$ is found to be

$$
E(N)=\frac{1-p_{1}^{k}}{\left(1-p_{1}\right) p_{1}^{k}+p_{2}\left(1-p_{1}^{k}\right)} .
$$

Corollary 2.3. In the special case $p_{2}=0, N$ has geometric distribution of order $k$ (see, e.g. Balakrishnan and Koutras (2002)).

If $X$ has a continuous phase type distribution with representation $P H_{c}(\boldsymbol{\alpha}, \mathbf{A})$, then its cumulative distribution function (cdf) is represented as

$$
F(t)=P(X \leq t)=1-\boldsymbol{\alpha} \exp (\mathbf{A} t) \mathbf{e}^{\prime}
$$

where the nonsingular matrix $A$ of dimension $k \times k$ has negative diagonal elements,and non-negative off-diagonal elements. Furthermore, all row sums of $A$ are non-positive. $\boldsymbol{\alpha}$ is substochastic vector; i.e. $\boldsymbol{\alpha} \mathbf{e}^{\prime} \leq 1$. The probability density function (pdf) of $X$ is given by

$$
f(t)=\boldsymbol{\alpha} \exp (\mathbf{A} t) \mathbf{a}^{0}
$$

where $\mathbf{a}^{0}=\mathbf{A e}^{\prime}$. The expected value of $X$ can be computed from $E(X)=$ $-\left(\boldsymbol{\alpha} \mathbf{A}^{-1} \mathbf{e}^{\prime}\right)$.

The proof of the following result can be found in He (2014).

Proposition 1. If $X_{1}, X_{2}, \ldots$ are independent and $X_{i} \sim P H_{c}(\boldsymbol{\alpha}, \mathbf{A}), i=1,2, \ldots$ and independently $N \sim P H_{d}(\boldsymbol{a}, \mathbf{Q})$. Then

$$
\sum_{i=1}^{N} X_{i} \sim P H_{c}\left(\boldsymbol{\alpha} \otimes \boldsymbol{a}, A \otimes \mathbf{I}+\left(\mathbf{a}^{0} \boldsymbol{\alpha}\right) \otimes \mathbf{Q}\right)
$$

where $\otimes$ is the Kronecker product.

\section{Optimal Replacement Time}

According to the classical age replacement policy, the system is replaced upon its failure or upon its reaching age $t$, whichever occurs first (Ahmad \& Kamaruddin, 2012). Let $c_{1}$ and $c_{2}$ denote respectively the costs of replacing non-failed and failed system. The mean cost rate per unit time as a function of the replacement age $t$ is given by

$$
C(t)=\frac{c_{1} P(T>t)+c_{2} P(T \leq t)}{E(\min (T, t))}=\frac{c_{2}+\left(c_{1}-c_{2}\right) P(T>t)}{E(\min (T, t))} .
$$


$E(\min (T, t))$ represent the average replacement time. Assuming that $T \sim P H_{c}(\boldsymbol{\alpha}, \mathbf{A})$, $P(T>t)$ and $E(\min (T, t))$ given in 3.1 are given respectively

$$
P(T>t)=\boldsymbol{\alpha} \mathbf{A}^{t} \mathbf{e}^{\prime}
$$

and

$$
E(\min (T, t))=\boldsymbol{\alpha} \mathbf{A}^{-1} \exp (\mathbf{A} t) \mathbf{e}^{\prime}-\boldsymbol{\alpha} \mathbf{A}^{-1} \mathbf{e}^{\prime}
$$

See, Eryilmaz (2017).

\section{Numerical Results}

Let $X_{i} \sim P H_{c}(\boldsymbol{\alpha}, \mathbf{A}), \boldsymbol{\alpha}=(0,1)$ and

$$
\mathbf{A}=\left[\begin{array}{cc}
-\lambda & 0 \\
\lambda & -\lambda
\end{array}\right]
$$

that is, the times between arrivals of shocks have Erlang distribution. In Figure 1, we plot the survival function of the system $P\{T>t\}$ for $p_{1}=0.4, p_{2}=0.1, k=2$ and $k=3$. As expected, the survival function of the system is increasing in $k$.

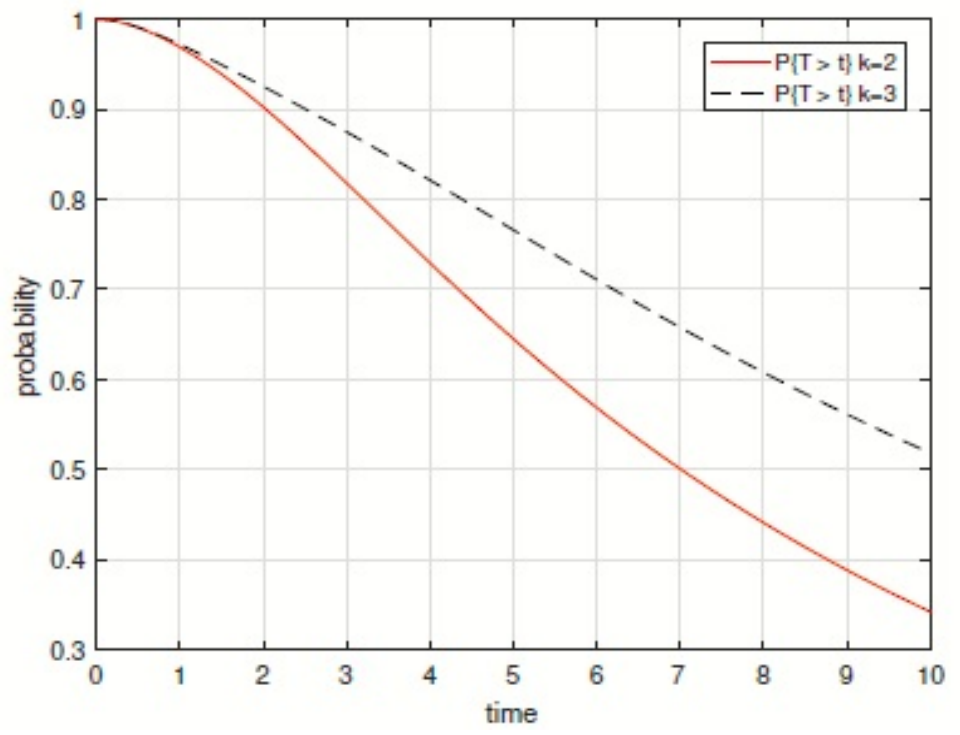

Figure 1. Survival functions under the two cases when $k=2$ and $k=3$.

Also, we compute mean time to failure of the system in Table 1 and plots the cost function of the system for $\lambda=1$. From Table 1, we observe that an increase in $k$ leads to an increase in mean time to failure of the system. With an increase in one of the parameters $p_{1}$ or $p_{2}$, the mean time to failure decreases. 
TABLe 1. Mean time to failure of the system

\begin{tabular}{llll}
\hline$k$ & $p_{1}$ & $p_{2}$ & $E(T)$ \\
\hline 2 & 0.3 & 0.1 & 11.818 \\
& 0.3 & 0.2 & 7.428 \\
& 0.4 & 0.1 & 9.334 \\
\hline 3 & 0.3 & 0.1 & 16.746 \\
& 0.3 & 0.2 & 9.114 \\
& 0.4 & 0.1 & 14.182
\end{tabular}

Figure 2 plots the cost function given by (3.1) when $c_{1}=1$ and $c_{2}=10$. As it is clear from Figure 2, the optimal replacement time is earlier for the case when $k=2$.

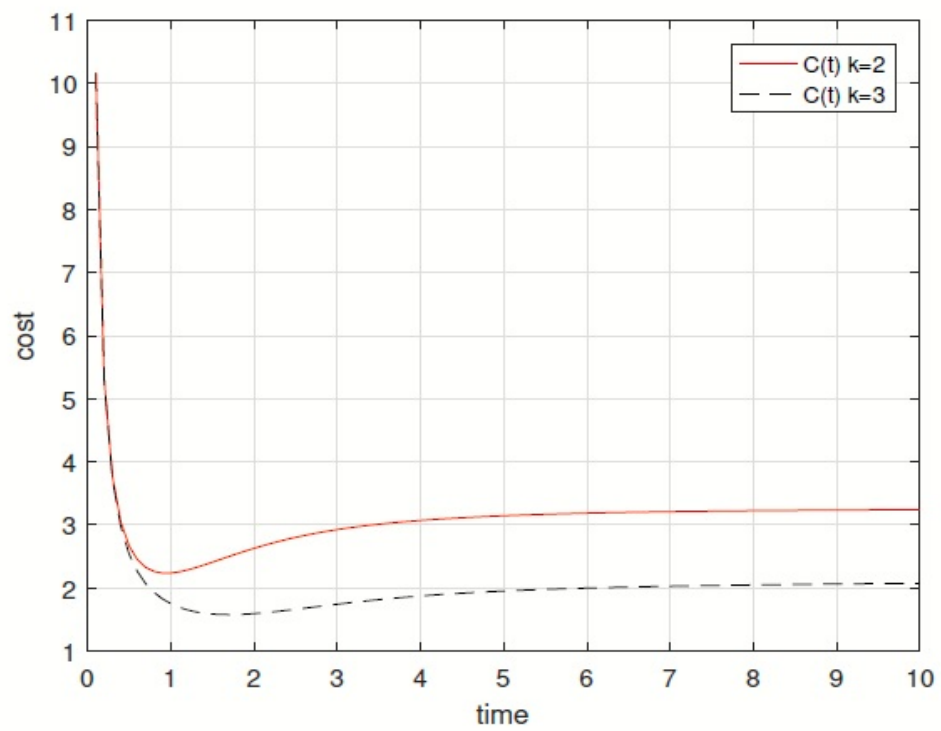

Figure 2. Cost functions for $c_{1}=1, c_{2}=10, p_{1}=0.6$ and $p_{2}=0.1$. 


\section{REFERENCES}

[1] Neuts, M. F., Meier, K. S. (1981). On the use of phase type distributions in reliability modelling of systems with two components. OR Spektrum, 2(4), 227-234.

[2] Ahmad R., Kamarudin S. (2012), An overview of time-based and condition-based maintenance in industrial application, Computers \& Industrial Engineering 63(1), 135-149.

[3] Gut, A.(2001) Mixed shock models, Bernoulli 7(3), 541-555.

[4] He, Q. M. (2014). Fundamentals of Matrix-Analytic Methods. NewYork: Springer, 22-43.

[5] Eryilmaz, S. (2017), Computing optimal replacement time and mean residual life in reliability shock models Computers \& Industrial Engineering, 103, 40-45.

[6] Eryilmaz, S. and Tekin, M. (2018). Reliability evaluation of a system under a mixed shock model, submitted for publication.

(Mustafa TEKIN) Gazi university, 06500, AnKara, Turkey

Current address: Gazi university, Graduate School Of Natural And Applied Sciences, 06500, Yenimahalle/ Ankara, Turkey

E-mail address, author one: mustafa.tekin@gazi.edu.tr

(Serkan ERYilmaZ) Atilim university, Department Of Industrial Engineering, 06836, ANKARA, TURKEY

E-mail address, author two: serkan.eryilmaz@atilim.edu.tr 